\title{
Information for genetic management of mtDNA disease: sampling pathogenic mtDNA mutants in the human germline and in placenta
}

\author{
D Marchington, ${ }^{1}$ S Malik, A Banerjee, ${ }^{2}$ K Turner, ${ }^{1}$ David Samuels, ${ }^{3}$ V Macaulay, ${ }^{4}$ \\ P Oakeshott, ${ }^{5}$ C Fratter, ${ }^{6}$ S Kennedy, ${ }^{1}$ J Poulton ${ }^{1}$
}

- Supplementary information is published online only. To view these files please visit the journal online http://jmg.bmi. com/content/vol47/issue4.

${ }^{1}$ Nuffield Department of Obstetrics, University of Oxford, The Women's Centre, Oxford, UK

${ }^{2}$ Department of Metabolic Medicine, Hammersmith Hospital NHS Trust, London, UK ${ }^{3}$ Center for Human Genetics Research, Department of Molecular Physiology and Biophysics, Vanderbilt University Medical Center, Nashville, Tennessee, USA

${ }^{4}$ Department of Statistics, University of Glasgow, Glasgow,

${ }^{5}$ Community Health Sciences, St George's Hospital Medical School, London, UK ${ }^{6}$ Oxford Medical Genetics Laboratory, Churchill Hospital, Oxford, UK

\section{Correspondence to}

Professor Joanna Poulton, Nuffield Department of Obstetrics, University of Oxford, The Women's Centre, Oxford OX3 9DU, UK; joanna.poulton@ obs-gyn.ox.ac.uk

Received 4 September 2009 Revised 21 October 2009 Accepted 22 October 2009

\section{ABSTRACT}

Background Families with a child who died of severe, maternally inherited mitochondrial DNA (mtDNA) disease need information on recurrence risk. Estimating this risk is difficult because of (a) heteroplasmy - the coexistence of mutant and normal mtDNA in the same person-and (b) the so-called mitochondrial bottleneck, whereby the small number of mtDNAs that become the founders for the offspring cause variation in dose of mutant mtDNA. The timing of the bottleneck and of segregation of mtDNA during foetal life determines the management options. Therefore, mtDNA heteroplasmy was studied in oocytes and placenta of women in affected families.

Results One mother of a child dying from Leigh syndrome due to the $9176 \mathrm{~T} \rightarrow \mathrm{C}$ mtDNA mutation transmitted various loads of mutant mtDNA to $\leq 3$ of 20 oocytes. This was used to estimate recurrence as $\leq 5 \%$. She subsequently conceived a healthy son naturally. Analysis of the placenta showed that some segregation also occurred during placental development, with the mutant $\mathrm{mtDNA}$ load varying by $>10 \%$ in a placenta carrying $65 \% 3243 \mathrm{~A} \rightarrow \mathrm{G}$ mutant mtDNA.

Discussion This is the first report of (a) an oocyte analysis for preconception counselling, specifically, refining recurrence risks of rare mutations and (b) a widely different load of a pathogenic mtDNA mutation in multiple oocytes, apparently confined to the germline, in an asymptomatic carrier of an mtDNA disease. This suggests that a major component of the bottleneck occurs during oogenesis, probably early in the foetal life of the mother. The variable mutant load in placenta implies that estimates based on a single sample in prenatal diagnosis of mtDNA disorders have limited accuracy.

Maternally inherited mitochondrial DNA (mtDNA) diseases are common genetic disorders, with the population prevalence of deafness associated with the $3243 \mathrm{~A} \rightarrow \mathrm{G}$ mtDNA mutation being 1 in $400{ }^{1}$ The more severe types often present in the neonatal period, with a range of devastating conditions, including cardiomyopathy, loss of vision and fatal liver disease. The severity of their expression varies because of heteroplasmy - the coexistence of mutant and normal mtDNA in the same person. A threshold effect (ie, tissues function normally unless the load of mutant mtDNA rises above a particular level) also exists in most mtDNA diseases, the progression of which is explained by preferential accumulation of mutant mtDNAs in affected tissues.

Couples who have had a child severely affected by a maternally inherited mtDNA disorder frequently ask about the much publicised nuclear transfer as a way of reducing transmission of mtDNA disease; however, this is not a realistic option at present. $^{2}$ They then request prenatal diagnosis in a subsequent pregnancy, which has not been widely applied to mtDNA disease. ${ }^{3}$ For instance, chorionic villus sampling (CVS) may be unreliable. ${ }^{4}$ High levels of mutant mtDNA in placental tissue indicate that the foetus is probably affected; zero levels indicate that it is probably unaffected, but intermediate levels ${ }^{5}$ are unhelpful. The correlation between mutant dose and disease severity is often poor. ${ }^{6-8}$

Providing accurate information about risk recurrence is similarly difficult, and so many families reluctantly decide not to have more children. Preconception counselling is problematic because of the mitochondrial "bottleneck". ${ }^{9-11}$ The most extreme example occurs after complete switching of mtDNA type in a single transmission, probably as a result of a mutation in a single mtDNA molecule, so that all a mother's mtDNA copies differ from those in her offspring by a single nucleotide. Significant mtDNA segregation occurs during oogenesis in both woman and mouse. $51012-15$ Furthermore, this may enable selection against detrimental mtDNA mutants in the mouse germline. $^{1617}$

Investigation of the bottleneck has had three foci. First, its exact timing and molecular basis remain controversial: the major component is held to occur at E7.5 in the mouse, ${ }^{13}$ early in oogenesis, while the mother is still herself a developing foetus. Second, the mutant load in the foetus may be determined by segregation between the blastocyst stage and fertilisation. This is because the majority of cells in the blastocyst will develop into extraembryonic structures, with perhaps as few as three cells of the inner cell mass developing into the embryo. Third, segregation of mutant mtDNA in extra-embryonic tissues would affect sampling of mtDNA at CVS. All of these are difficult to investigate in humans, but are clearly critical in advising families about their options. ${ }^{5}$

The current investigation of the mtDNA bottleneck is highly relevant because it significantly amplifies the existing dataset in humans. First, we have studied the segregation of both pathogenic and non-functional polymorphic mtDNA variants in the germline. Two women who sought preconception counselling enabled us to study two pathogenic mutants in their oocytes. Hence, we have documented a unique case of germline mosaicism 
for an mtDNA mutant. Second, we have investigated segregation after conception in a placenta from a woman who has mitochondrially inherited diabetes and deafness due to the pathogenic mtDNA $3243 \mathrm{~A} \rightarrow \mathrm{G}$ mutant. We documented the segregation of mtDNA subpopulations, with estimates of heteroplasmic load varying by as much as $10 \%$ in different samples.

We conclude that a major component of the mitochondrial bottleneck has occurred by the time oocytes are mature, and that segregation, and potentially selection, may continue post conception. Oocyte sampling is useful for genetic counselling. Our data support the use of prenatal diagnosis with certain provisos.

\section{MATERIALS AND METHODS}

Informed consent was obtained from subjects (or their guardians), and ethics committee approval was from the Oxford Research Ethics Committee.

\section{Patients and oocyte sampling}

Case 1

The woman's first child, who died aged 4 years, had developmental regression, lactic acidosis, cardiomyopathy and MRI changes consistent with maternally inherited Leigh disease. An mtDNA mutation in the child was identified at 9176T $\rightarrow$ C. In this disorder, the published data suggest that individuals carrying less than $80-90 \%$ mutant mtDNA are asymptomatic. ${ }^{18}$ However, 9176 $\rightarrow \mathrm{C}$ mutant mtDNA was undetectable in the mother's blood, buccal smear and urinary epithelial cells. She opted for oocyte sampling using conventional in vitro fertilisation methods because of the uncertainty surrounding the recurrence risk. Twenty oocytes were retrieved under ultrasound control following gonadotrophin stimulation, lysed and amplified by PCR as before.

\section{Case 2}

The woman's first child died aged 21 months with liver disease, lactic acidosis and anaemia, which are characteristics of Pearson syndrome. She had been given ciprofloxacin around the time of conception, a drug known to cause mtDNA depletion in cell lines but never previously implicated in the pathogenesis of mtDNA rearrangements. Pearson syndrome is usually sporadic; however, maternal transmission was considered possible in this case because the affected child had duplications as well as deletions (rearrangements) of mtDNA, and these may be maternally inherited. ${ }^{4}{ }^{19}$ Furthermore, the mother had a cardiac conduction defect, and these can be caused by mtDNA rearrangements. No rearranged mtDNA was detectable in the mother's blood; however, maternal muscle was not available for analysis. The parents requested oocyte sampling even though the recurrence risk was believed to be low $\left(\leq 5 \%{ }^{40}\right)$ because they could not contemplate the possibility of another affected child. Gonadotrophin stimulation and oocyte collection were carried out as above. Seventeen oocytes were collected (see supplementary information for the detailed analytical method).

\section{Placental donor: case 3}

A known patient with diabetes and deafness due to the mtDNA $3243 \mathrm{~A} \rightarrow \mathrm{G}$ mutation, who has one apparently healthy child, became pregnant for the seventh time at age 40 years. Weight gain was poor and insulin requirement barely increased. Following pre-eclampsia, which was a feature of all her pregnancies and an emerging complication of this mutation, ${ }^{21}$ she gave birth to a severely growth-restricted stillborn foetus weighing only $350 \mathrm{~g}$. About $10 \%$ of the foetal component of her $30 \mathrm{~g}$ placenta was frozen for analysis.

\section{Isolation of DNA from single oocytes}

Detailed methods for isolating oocytes and extracting DNA are in supplementary information B.

\section{PCR analysis of oocytes and placenta (see supplementary information B)}

The $9176 \mathrm{~T} \rightarrow \mathrm{C}$ mutation was quantitated using standard methods. ${ }^{22}{ }^{23}$ Last-cycle labelling with ${ }^{32}$ P-dCTP and Phosphorimager analysis were used. The sensitivity of the method was generally high, at least $2 \%$ 9176T $\rightarrow \mathrm{C}$ mtDNA being detectable on a wild-type background (supplementary information $\mathrm{D}$ figure 2). The $3243 \mathrm{~A} \rightarrow \mathrm{G}$ mutation was quantitated using last-cycle fluorescent labelling followed by capillary electrophoresis on $\mathrm{ABI} 3100,{ }^{22} 23$ the sensitivity being $<2 \%$.

The method for estimating risk used a mathematical model of the bottleneck with a range of bottleneck sizes ${ }^{9}$ (Supplementary information A).

To detect mtDNA carrying the rearrangement, primers that straddled the deletion junction were used as previously shown. ${ }^{24}$ The sensitivity of the method was again high $(1 \%$ could be detected on a wild-type background; data not shown).

To distinguish between maternal and foetal DNA in the mtDNA $3243 \mathrm{~A} \rightarrow \mathrm{G}$ placenta, we carried out microsatellite analysis using the AmpflSTR Profiler Plus kit (Applied Biosystems, Foster City, California, USA).

\section{Statistical analysis}

Analysis of the bottleneck in human oocytes used a binomial sampling distribution and is outlined in Supplementary information A. The mtDNA mutant in individual oocytes was compared with the Kimura distribution as previously shown 25 (Supplementary information). Differences in mtDNA 3243A $\rightarrow$ G mutant load in placenta were assessed by using $t$ test.

\section{RESULTS: 00CYTE STUDY}

We aimed to study the mtDNA bottleneck by determining the levels of mutant mtDNA in oocytes from two mothers of affected children, to refine the recurrence risks for subsequent pregnancies.

\section{Case 1: maternally inherited Leigh disease due to $9176 \mathrm{~T} \rightarrow \mathrm{C}$} Figure 1 shows that levels of $\leq 80 \%$ 9176 $\rightarrow \mathrm{C} \mathrm{mtDNA}$ were detected in $\leq 3$ of the 20 oocytes obtained. Calculation of recurrence risk is complex, with two sources of uncertainty. One is of the actual load of mutant mtDNA molecules in the germline, given the observations and the accuracy of the quantitation. The other is the probability that any particular oocyte will develop into an individual with a mutant load above the disease threshold (taken as $80 \%$ for $9176 \mathrm{~T} \rightarrow \mathrm{C}$ ). ${ }^{18}{ }^{22} 26-31 \mathrm{We}$ estimated the recurrence risk to be $\leq 5 \%$; the subsequent more detailed analysis suggests that it is $\leq 3 \%$ (Supplementary information). The distribution of the mutant load of mtDNA was compared to the Kimura distribution. It was consistent with the theoretical distribution (Supplementary information) with an unusually high variance, twice as high as the heteroplasmy variance in oocytes that was previously reported. ${ }^{32}$ The woman subsequently decided to conceive naturally, and happily ${ }^{3}$ delivered a healthy baby at term. CVS was carried out as in a previous family ${ }^{18}$ and mutant mtDNA was undetectable at 10 weeks of gestation, in 16 different samples of term placenta and in cord blood. 
Figure 1 Detection of the $9176 \mathrm{~T} \rightarrow \mathrm{C}$ mutation in 20 oocytes after superovulation, in the mother's blood and in muscle from the affected child who died. The 9176T $\rightarrow C$ mutant mitochondrial DNA (mtDNA) generates a new restriction site, so that, in the affected child, the upper wild-type (normal) band is replaced by a lower (mutant) fragment. The child carried 99\% $9176 \mathrm{~T} \rightarrow \mathrm{C}$ mutant $\mathrm{mtDNA}$ in all tissues examined (marked "affected"), but none was detectable in the mother's blood, CVS, placenta or cord blood from her foetus. Oocyte 17 (mutant band judged just visible) carried $\leq 5 \% 9176 \mathrm{~T} \rightarrow \mathrm{C}$ mutant mtDNA. Oocytes 12 and 13 could not be dissected apart and carried $40 \%$ mutant mtDNA, consistent with $40-80 \%$ in one and $0-40 \%$ mutant in the other. The PCR efficiency was too low to be used in two oocytes (No 9 and 11) and these were excluded from the analysis. Assuming a mutant mtDNA load of $80 \%$ in oocyte $12,0 \%$ in oocyte 13 and $5 \%$ in oocyte 17 , the average load in the 18 useable oocytes was $5 \%$. Assuming a threshold mutant load of $80 \%$ for symptomatic disease and taking into account a range of bottlenecks, the estimated recurrence risk was $\leq 5 \%$ (supplementary information).

\section{Case 2 Pearson syndrome due to mtDNA rearrangement}

No rearranged mtDNA was detected in any of the 10/17 oocytes from which PCR product was obtained. The recurrence risk was estimated as $<1 \%$ (Supplementary information A). She has not yet conceived.

\section{RESULTS: PLACENTAL STUDY}

DNA from 10 samples of the mtDNA $3243 \mathrm{~A} \rightarrow$ G placenta, each approximately $10 \mathrm{mg}$, were analysed for both the mutant load and mtDNA copy number as shown in figure 2 . The mother's mtDNA $3243 \mathrm{~A} \rightarrow \mathrm{G}$ mutant load was $24 \%$ in blood DNA. The percentage mutant ranged from $55 \%$ to $64 \%$ and the mtDNA copy number varied almost fivefold (supplementary figure). To assess the reproducibility of these differences, the samples containing the highest and lowest mutant loads were rerun (including PCR and restriction digestion) in quadruplicate (each one being loaded in duplicate) on two further occasions. The average mutant loads differed by $11.95 \%$ (being $49.41 \%(0.42 \%)$ and $61.42 \%(0.42 \%), p<0.0025$, $t$ test $)$, confirming that this reflects a real difference in mutant load in different regions of the same placenta. Microsatellite analysis of all placental samples (using the AmpflSTR Profiler Plus kit, Applied Biosystems) showed that all samples were entirely composed of foetal and not maternal DNA.

\section{DISCUSSION}

These data significantly increase the data demonstrating segregation of pathogenic mutants in the human germline and in embryonic development. We have successfully used oocyte sampling to predict patient-specific recurrence risk for preconception counselling in heteroplasmic mtDNA disorders. Our demonstration of a widely different load of a pathogenic mtDNA mutation in multiple oocytes, apparently confined to the germline in an asymptomatic carrier of an mtDNA disease, demonstrates that segregation occurs during oogenesis. We have shown variation in the load of pathogenic mtDNA between small placental samples. In agreement with previous studies, ${ }^{9} 131517$ our findings suggest that much of the segregation for these mtDNA variants, known as the mitochondrial bottleneck, occurs during oocyte development, with additional mtDNA segregation during differentiation of embryo and extra-embryonic structures.

As far as we know, this is the first report of the use of oocyte sampling to predict patient-specific recurrence risk for these disorders. Recurrence estimates given to the mothers were $\leq 5 \%$ and $<1 \%$. The new risk estimations enabled both women, who had decided against conceiving again, to change their minds This is also the first demonstration of an mtDNA mutation in multiple oocytes, apparently confined to the germline, in an asymptomatic carrier of an mtDNA disease. As the $9176 \mathrm{~T} \rightarrow \mathrm{C}$
Figure 2 Proportion of $3243 \mathrm{~A} \rightarrow \mathrm{G}$ mutant mitochondrial DNA (mtDNA) differs by over $10 \%$ in 10 different samples of human placenta.

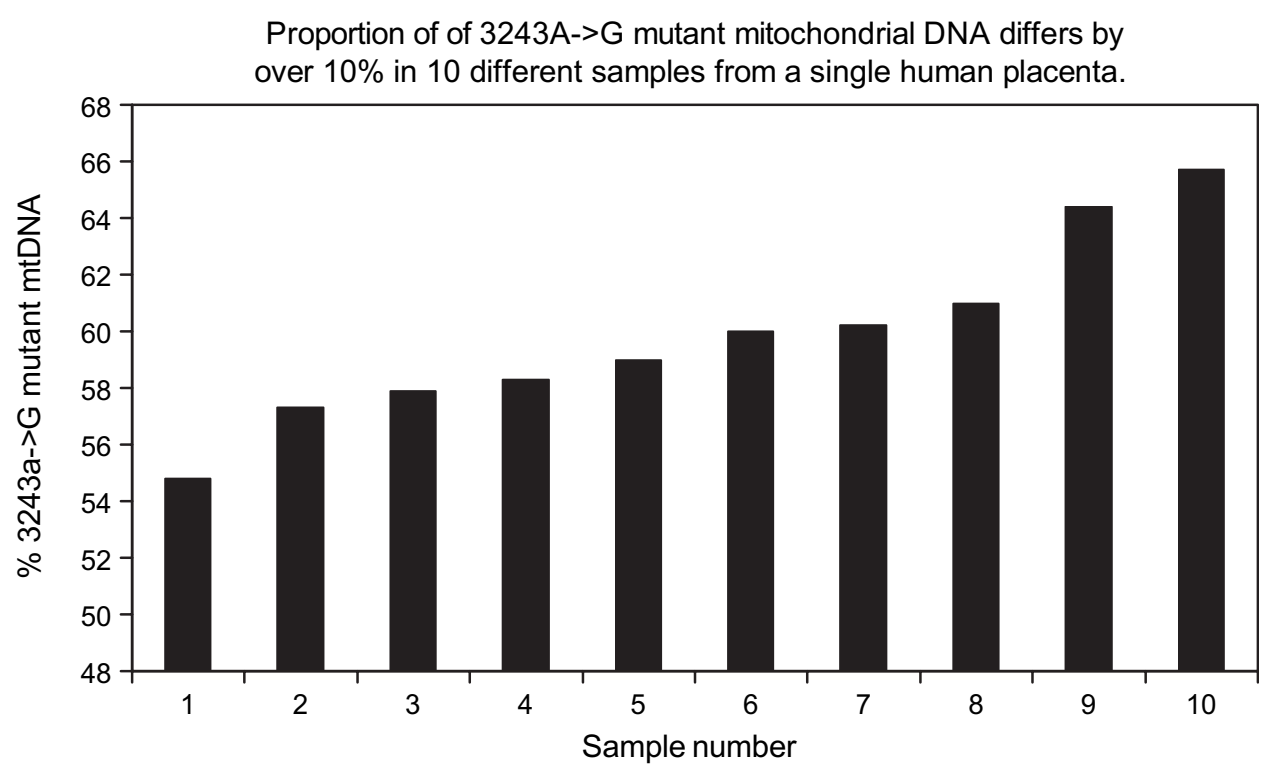


mtDNA mutation was confined to between one and three oocytes, it presumably arose within the germline during the foetal life of the mother. This would be consistent with the high heteroplasmy variance (thus, small number of mtDNA founders) identified in the statistical analysis (supplementary information). The mutation could have been initially present but selected out of other tissues such as blood, or at a level below the detection limit. In case 2 , there was no evidence of transmission of rearranged mtDNA to the oocytes, and a work that was unpublished at the time ${ }^{33}$ supports our advice that she had a low recurrence.

A limitation of using oocyte sampling for estimating recurrence risks is the scanty literature correlating the load of mutant mtDNA and disease severity. Individuals carrying a mutant load of $<80 \% 9176 \mathrm{~T} \rightarrow \mathrm{C}$ mutant mtDNA in blood mtDNA have minimal problems ${ }^{18}$ but many mtDNA disorders do not have clearly defined thresholds, which limits the accuracy of recurrence risk estimations. In particular, prenatal diagnosis has limited application in most families with Leber hereditary optic neuropathy, because these tend to be homoplasmic with a low penetrance of the disease phenotype: where they are heteroplasmic, there is a substantial risk of developing visual impairment over a wide range of mutant loads. ${ }^{34}$ Clinical geneticists have performed preimplantation genetic diagnosis (PGD) in these families, selecting embryos for female sex as women have a substantially lower risk of developing symptoms than men. ${ }^{35}$ We cannot be sure that the mutant load in oocytes precisely reflects that in live born children. However, the load in oocytes is likely to be more relevant than the load in maternal muscle and has some support from animal studies. ${ }^{36}$

Segregation of the $3243 \mathrm{~A} \rightarrow \mathrm{G}$ mtDNA in the placenta showed that mtDNA mutant load differed across the placenta by as much as $10 \%$, and this was not due to maternal contamination. In addition, mtDNA copy number varied approximately fivefold (see online figure 3). Similarly, we saw a statistically significant variation of similar magnitude in the distribution of one of two polymorphic variants, consistent with previous data. ${ }^{37} \mathrm{We}$ previously demonstrated that the average level of polymorphic heteroplasmic mtDNA variants is very similar in mother, cord blood and placenta. However, where placental samples were very small $(<10 \mathrm{mg})$, there was some evidence of variation in the distribution of mtDNA polymorphic variants. Differences in mtDNA content of the different cell types present in the 10 samples of $3243 \mathrm{~A} \rightarrow \mathrm{G}$ placenta might underlie the fivefold difference in mtDNA copy number that we identified. Indeed, we have documented a similar degree of variation in mtDNA content in control mouse placenta (Sajida Malik and Jo Poulton, unpublished data 2008). However, variation of both mutant load and mtDNA copy number is a well-known characteristic of the $3243 \mathrm{~A} \rightarrow \mathrm{G}$ mtDNA mutant, resulting in "ragged red" fibres in skeletal muscle probably due to a combination of random drift ${ }^{38}$ and compensation for the mitochondrial dysfunction. ${ }^{39}$ The magnitude of the amplification of mtDNA copy number is more comparable to that seen in ragged red fibres in skeletal muscle ${ }^{40}$ than tissue culture (see online figure 3). As this mother was prone to pre-eclampsia, the placenta may have been under considerable oxidative stress quite apart from the $3243 \mathrm{~A} \rightarrow \mathrm{G}$ mtDNA mutant. We conclude that we documented variation in mtDNA content in different regions of a single placenta, and that oxidative stress and/or variable mitochondrial function may contribute to this.

The current study supports our previous suggestion that a single sample at CVS, or indeed PGD, may be insufficient to assess the mutant load. In preliminary data from human blas- tomeres $^{41}{ }^{42}$ (supplementary information 2), we provide some support for earlier findings from mouse ${ }^{43}$ and human ${ }^{44}$ that heteroplasmy in blastomeres varies more between embryos than within embryos. Nevertheless, in the absence of extensive data, it may be necessary to take two samples at $\mathrm{CVS}^{37}$ because of the considerable variation in the level of mtDNA mutant that we documented in placental patches. This also argues for repeated sampling at PGD, say two separate blastomeres. However, taking two samples would likely reduce the viability of the embryo. Another less invasive possibility would be to sample a blastomere and the cytoplasm surrounding a polar body, as preliminary data from mouse ${ }^{43}$ suggest that the mutant load is identical. As an alternative strategy, ${ }^{45}$ initially a single blastomere would be sampled from each embryo to identify those with low levels of mutant mtDNA. All blastomeres from the unsuitable embryos would then be analysed to ensure that the distribution of mutants is uniform within embryos. Only if this was reassuring would an embryo with a low level of mutant mtDNA be implanted.

The management options in clinical practice are extremely complicated. ${ }^{3}$ It is therefore very taxing and ethically challenging to advise women what to do. It is especially difficult for geneticists to estimate recurrence risk in heteroplasmic mtDNA diseases. In these disorders, prenatal diagnosis based on respiratory chain enzyme activities is unreliable, ${ }^{46}$ and that based on mtDNA is complex (because of the problem of heteroplasmy). PGD, which involves sampling blastomeres from early embryos and only transferring low-risk ones, could potentially avoid this dilemma. This procedure is hard to apply to women whose oocytes all have an unacceptably high mutant load. While there are scanty published reports of this technique for mtDNA disease, $^{5} 414247$ recent mouse studies suggest that strong selection in the germline against certain types of mutation ${ }^{16} 17$ may increase the chance of identifying embryos with an acceptably low mutant load. More data are needed to determine whether there is selection against the $3243 \mathrm{~A} \rightarrow \mathrm{G}$ mutation in the human germline. Other authors discuss more radical measures such as replacing the zygote's mutant mitochondria with wild type. ${ }^{18} 444849$

In conclusion, despite the uncertainties, two women willingly accepted oocyte sampling to estimate their risk, and one has since had a healthy child who is now aged 5 years old. We feel that undergoing two procedures is justified by the problems attributable to heteroplasmy. We advocate that clinicians consider whether taking two samples at CVS may be appropriate in specific cases. Furthermore, interpretation of CVS may be much more feasible where PGD has been used to select suitable embryos. The positive outcome of oocyte sampling has important practical and theoretical implications for genetic counselling of families affected by devastating mtDNA disorders. Even though we have demonstrated a major component of the bottleneck occurring during oogenesis, an essential precondition for PGD, our analysis also suggests that a considerable variation may develop during the later stages of placental development. It may therefore be advisable to take two samples at CVS in selected cases. These studies are generally encouraging for prenatal diagnosis in selected mtDNA diseases.

Acknowledgements We thank the patients, their families and the physicians fo their help, particularly Drs A Dornhurst and J Wyatt-Ashmead. We are grateful to Professor DH Barlow for initiating this project and to Dr Anneke Seller for provision of laboratory facilities, Julie Evans and Passorn Wonnapinij for technical support, Mrs Annie Shrier and Dr J Marchington for critical appraisal of the manuscript and Dr J Birks for statistical advice. This work was supported by Action Medical Research, the Wellcome Trust, the Medical Research Council, the Royal Society and 
the Oxford Partnership Comprehensive Biomedical Research Centre with funding from the Department of Health's National Institute for Health Research Biomedical Research Centres funding scheme. The views expressed in this publication are those of the authors and not necessarily those of the Department of Health.

Funding Action Medical Research. Other funders: Wellcome Trust; Medical Research Council, Royal Society and Department of Health's National Institute for Health Research

\section{Competing interests None}

Ethics approval This study was conducted with the approval of the Oxford Research Ethics Committee.

Patient consent Obtained.

Provenance and peer review Not commissioned; externally peer reviewed.

\section{REFERENCES}

1. Manwaring N, Jones MM, Wang JJ, Rochtchina E, Howard C, Mitchell P, Sue CM. Population prevalence of the MELAS A3243G mutation. Mitochondrion 2007:7:230-3.

2. BBC News Channel. Three-parent embryo formed in lab. 2 May 2008. http://news. bbc.co.uk/1/hi/7227861.stm (accessed February 2010).

3. Poulton J, Kennedy S, Oakeshott P, Wells D. Preventing transmission of maternally inherited mitochondrial DNA diseases. BMJ 2009;338:345-9.

4. Poulton J, Turnbull DM. 74th ENMC international workshop: mitochondrial diseases 19-20 November 1999, Naarden, The Netherlands. Neuromuscul Disord 2000;10:460-2

5. Bouchet C. Steffann J Corcos J Monnot S, Paquis V, Rotig A, Lebon S, Levy P, Royer G, Giurgea I, Gigarel N, Benachi A, Dumez Y, Munnich A, Bonnefont JP. Prenatal diagnosis of MELAS syndrome: contribution to understanding mitochondrial DNA segregation during human embryo fetal development. J Med Genet 2006;43:788-92

6. Rahman S, Poulton J, Marchington D, Suomalainen A. Decrease of 3243 $A \rightarrow G$ mtDNA mutation from blood in MELAS syndrome: a longitudinal study. Am J Hum Genet 2001;68:238-40.

7. White S, Shanske S, McGill J, Mountain H, Geraghty M, DiMauro S, Dahl H Thorburn D. Mitochondrial DNA mutations at nucleotide 8993 show a lack of tissueor age-related variation. J Inherit Metab Dis 1999;22:899-914.

8. White SL, Shanske S, Biros I, Warwick L, Dahl HM, Thorburn DR, Di Mauro S. Two cases of prenatal analysis for the pathogenic $T$ to $G$ substitution at nucleotide 8993 in mitochondrial DNA. Prenat Diagn 1999;19:1165-8.

9. Marchington D, Hartshorne G, Barlow D, Poulton J. Evidence from human oocytes for a genetic bottleneck in a mitochondrial DNA disease. Am J Hum Genet 1998:63:769-75

10. Poulton J, Macaulay V, Marchington D. Transmission, genetic counselling and prenatal diagnosis of mitochondrial DNA disease. In: Holt I, ed. Genetics of mitochondrial disease. Oxford: Oxford University Press, 2003:309-26.

11. Thorburn DR, Dahl HH. Mitochondrial disorders: genetics, counseling, prenatal diagnosis and reproductive options. Am J Med Genet 2001;106:102-14.

12. Chinnery PF, Howell N, Lightowlers RN, Turnbull DM. Genetic counselling and prenatal diagnosis for mtDNA disease. Am J Hum Genet 1998;63:1908-11. [letter; comment]

13. Cree LM, Samuels DC, de Sousa Lopes SC, Rajasimha HK, Wonnapinij P, Mann JR, Dahl $\mathrm{HH}$, Chinnery PF. A reduction of mitochondrial DNA molecules during embryogenesis explains the rapid segregation of genotypes. Nat Genet 2008:40:249-54.

14. Cao L, Shitara H, Horii T, Nagao Y, Imai H, Abe K, Hara T, Hayashi J, Yonekawa H. The mitochondrial bottleneck occurs without reduction of mtDNA content in female mouse germ cells. Nat Genet 2007:39:386-90.

15. Marchington D, Hartshorne G, Barlow D, Poulton J. Homoploymeric tract heteroplasmy in mtDNA from tissues and single oocytes: support for a genetic bottleneck. Am J Hum Genet 1997:60:408-16.

16. Stewart JB, Freyer C, Elson JL, Wredenberg A, Cansu Z, Trifunovic A, Larsson NG. Strong purifying selection in transmission of mammalian mitochondrial DNA. PLOS Biol 2008; 6 :e10.

17. Fan W, Waymire KG, Narula N, Li P, Rocher C, Coskun PE, Vannan MA, Narula J, Macgregor GR, Wallace DC. A mouse model of mitochondrial disease reveals germline selection against severe mtDNA mutations. Science 2008;319:958-62.

18. Jacobs LJ, de Coo IF, Nijland JG, Galjaard RJ, Los FJ, Schoonderwoerd K, Niermeijer MF, Geraedts JP, Scholte HR, Smeets HJ. Transmission and prenatal diagnosis of the T9176C mitochondrial DNA mutation. Mol Hum Reprod 2005;11:223-8.

19. Poulton J, Holt I. Mitochondrial DNA: does more lead to less? Nat Genet 1994; : :313-15

20. Poulton J, Marchington DR. Progress in genetic counselling and prenatal diagnosis of maternally inherited mtDNA diseases. Neuromuscul Disord 2000:10:484-7.

21. Turnbull H, Whittaker R, Phillips A, Poulton J, Turnbull D, McFarland R, eds. The risk of complications of pregnancy and labour for women with mitochondrial disease Euromit7. Journal of Internal Medicine. Stockholm, Sweden: Journal of Internal Medicine, 2008.

22. Thyagarajan D, Shanske S, Vazquez-Memije M, De Vivo D, Di Mauro S. A novel mitochondrial ATPase 6 point mutation in familial bilateral striatal necrosis. Ann Neurol 1995; 38:468-72.
23. White HE DV, Seller A, Fratter C, Harvey JF, Cross NC. Accurate detection and quantitation of heteroplasmic mitochondrial point mutations by pyrosequencing. Genet Test 2005:9:190-9.

24. Hamblet NS, Castora FJ. Mitochondrial DNA deletion analysis: a comparison of PCR quantitative methods. Biochem Biophys Res Commun 1995;207:839-47.

25. Wonnapinij $\mathbf{P}$, Chinnery PF, Samuels DC. The distribution of mitochondrial DNA heteroplasmy due to random genetic drift. Am J Hum Genet 2008;83:582-93.

26. Dionisi-Vici C, Seneca S, Zeviani M, Fariello G, Rimoldi M, Bertini E, De Meirleir L. Fulminant Leigh syndrome and sudden unexpected death in a family with the T9176C mutation of the mitochondrial ATPase 6 gene. J Inherit Metab Dis 1998;21:2-8.

27. Campos Y, Martin MA, Rubio JC, Solana LG, Garcia-Benayas C, Terradas JL, Arenas J. Leigh syndrome associated with the T9176C mutation in the ATPase 6 gene of mitochondrial DNA. Neurology 1997:49:595-7.

28. Makino M, Horai S, Goto Y, Nonaka I. Confirmation that a T-to-C mutation at 9176 in mitochondrial DNA is an additional candidate mutation for Leigh's syndrome. Neuromuscul Disord 1998:8:149-51.

29. Makino M, Horai S, Goto Y, Nonaka I. Mitochondrial DNA mutations in Leigh syndrome and their phylogenetic implications. J Hum Genet 2000;45:69-75

30. Wilson CJ, Wood NW, Leonard JV, Surtees R, Rahman S. Mitochondrial DNA point mutation T9176C in Leigh syndrome. J Child Neurol 2000;15:830-3

31. Carrozzo R, Rizza T, Lucioli S, Pierini R, Bertini E, Santorelli FM. A mitochondrial ATPase 6 mutation is associated with Leigh syndrome in a family and affects proton flow and adenosine triphosphate output when modeled in Escherichia coli. Acta Paediatr Supp/ 2004;93:65-7.

32. Brown DT, Samuels DC, Michael EM, Turnbull DM, Chinnery PF. Random genetic drift determines the level of mutant mtDNA in human primary oocytes. Am J Hum Genet 2001:68:533-6.

33. Chinnery PF, DiMauro S, Shanske S, Schon EA, Zeviani M, Mariotti C, Carrara F, Lombes A, Laforet P, Ogier $H$, Jaksch M, Lochmuller $H$, Horvath R, Deschauer M, Thorburn DR, Bindoff LA, Poulton J, Taylor RW, Matthews JN, Turnbull DM. Risk of developing a mitochondrial DNA deletion disorder. Lancet 2004;364:592-6.

34. Black GC, Morten K, Laborde A, Poulton J. Leber's hereditary optic neuropathy: heteroplasmy is likely to be significant in the expression of LHON in families with the 3460 ND1 mutation. Br J Ophthalmol 1996;80:915-17.

35. Black G, Craig I, Oostra R, Norby S, Rosenberg T, Morten K, Laborde A, Poulton J. Leber's hereditary optic neuropathy: implications of the sex ratio for linkage studies in families with the 3460 ND1 mutation. Eye 1995;9:513-16.

36. Jenuth J, Peterson A, Fu K, Shoubridge E. Random genetic drift in the female germline explains the rapid segregation of mammalian mitochondrial DNA. Nat Genet 1996:14:146-51

37. Marchington D, Scott-Brown M, Barlow D, Poulton J. Moscaicism for mitochondria DNA polymorphic variants in placenta has implications for the feasibility of prenatal diagnosis in mtDNA diseases. Eur J Hum Genet 2006;14:816-23.

38. Chinnery PF, Samuels DC. Relaxed replication of mtDNA: a model with implications for the expression of disease. Am J Hum Genet 1999;64:1158-65.

39. Moreno-Loshuertos R, Acin-Perez R, Fernandez-Silva P, Movilla N, Perez-Martos A de Cordoba SR, Gallardo ME, Enriquez JA. Differences in reactive oxygen species production explain the phenotypes associated with common mouse mitochondrial DNA variants. Nat Genet 2006:38:1261-8.

40. Bentlage HA, Attardi G. Relationship of genotype to phenotype in fibroblast-derived transmitochondrial cell lines carrying the 3243 mutation associated with the MELAS encephalomyopathy: shift towards mutant genotype and role of mtDNA copy number. Hum Mol Genet 1996;5:197-205

41. Monnot S, Gigarel N, Hesters L, Burlet P, Benachi A, Dumez Y, Tachdjian G, Rötig A Frydman R, Munnich A, Frydman N, Bonnefont J, Steffann J, eds. The challenge of prenatal and preimplantation genetic diagnosis of mitochondrial DNA disorders. European Society of Human Genetics Annual Meeting, 2009.

42. de Die-Smulders C, Smeets $\mathrm{H}$, eds. The challenge of prenatal and preimplantation genetic diagnosis of mitochondrial disorders. European Society of Human Genetics Annual Meeting, 2009.

43. Dean NL, Battersby BJ, Ao A, Gosden RG, Tan SL, Shoubridge EA. Prospect of preimplantation genetic diagnosis for heritable mitochondrial DNA diseases. Mol Hum Reprod 2003:9:631-8.

44. Steffann J, Frydman N, Gigarel N, Burlet P, Ray PF, Fanchin R, Feyereisen E, Kerbrat V, Tachdjian G, Bonnefont JP, Frydman R, Munnich A. Analysis of mtDNA variant segregation during early human embryonic development: a tool for successful NARP preimplantation diagnosis. J Med Genet 2006;43:244-7.

45. Bredenoord A, Dondorp W, Pennings G, De Die-Smulders C, Smeets H, De Wert G. PGD to reduce reproductive risk: the case of mitochondrial DNA disorders. Hum Reprod 2008;23:2392-401.

46. Niers L, van den Heuvel L, Trïbels F, Sengers R, Smeitink J. Prerequisites and strategies for prenatal diagnosis of respiratory chain deficiency in chorionic villi. $J$ Inherit Metab Dis 2003;26:647-58.

47. Steffan J, Gigarel N, Frydman N, Burlet P, Bonnefort J-P, Ray P, Kerbrat V, Tachdjian G, Frydman R, Munnich A. Preimplantation diagnosis of the NARP mitochondrial DNA mutation. Reprod Biomed Online 2005;10(Suppl 2):9.

48. Jacobs LJ, de Wert G, Geraedts JP, de Coo IF, Smeets HJ. The transmission of OXPHOS disease and methods to prevent this. Hum Reprod Update 2006:12:119-36.

49. Poulton J, Kennedy S, Oakeshott P, St John J. Nuclear transfer to prevent mitochondrial DNA diseases. Lancet 2006;368:841. 